\title{
TESTE DE HETEROGENEIDADE PARA MINÉRIO DE ALUMÍNIO
}

\author{
D. A BORTOLETO', A CHIEREGATI', R. L. MACIEL ${ }^{2}$ e A. H. PEREIRA ${ }^{2}$ \\ ${ }^{1}$ Departamento de Engenharia de Minas e Petróleo - Universidade de São Paulo \\ bortoda@usp.br - ana.chieregati@poli.usp.br \\ ${ }^{2}$ Alcoa World Alumina - Juruti-PA \\ railson.maciel@alcoa.com.br - antonio.h.pereira@alcoa.com.br
}

Artigo submetido em novembro/2013 e aceito em janeiro/2014

DOI: http://dx.doi.org/10.15628/holos.2014.1750

\section{RESUMO}

A amostragem pode ser definida como o conjunto de operações destinadas à obtenção de uma amostra representativa de uma dada população ou lote de material. A redução da massa deve ser feita de forma criteriosa a fim de assegurar a confiabilidade dos processos envolvidos, não alterando as propriedades físico-químicas, tais como, teor e constituintes mineralógicos, do lote inicial. Mesmo que todos os procedimentos de amostragem sejam corretamente realizados, ainda haverá um erro associado à heterogeneidade constitucional do minério que não pode ser eliminado; o erro fundamental de amostragem (FSE). Neste contexto, os testes de heterogeneidade foram desenvolvidos com o objetivo de estimar o erro fundamental de amostragem. O presente trabalho estuda o desvio padrão gerado pela heterogeneidade constitucional do minério de alumínio da região oeste do Pará, a partir dos resultados obtidos no teste de heterogeneidade, visando otimizar protocolos de amostragem

PALAVRAS-CHAVE: amostragem, minério de alumínio, erro fundamental

\section{HETEROGENEITY TEST FOR ALUMINIUM ORE FROM NORTHERN BRAZIL}

\section{ABSTRACT}

Sampling can be defined as a series of operations aimed to obtaining a representative sample of a given population or bulk material, where the mass reduction must be done carefully to ensure reliability of the processes involved. Even if all sampling procedures are properly carried out, there is still an error associated with the heterogeneity of the deposit that can never be eliminated, the fundamental sampling error
Consequently each sampling stage will generate an error which must be known for the quality control results to be reliable. Heterogeneity tests were developed with the objective of estimating the fundamental sampling error. This paper studies the standard deviation generated by the constitutional heterogeneity of the aluminum ore from northern Brasil aiming to optimize sampling protocols.

KEYWORDS: sampling, aluminum ore, fundamental sampling error. 


\section{INTRODUÇÃO}

Para determinar o desempenho operacional de um processo de tratamento de minérios é essencial o conhecimento das características físico-químicas do produto. Esta determinação para uma dada propriedade ou característica do material só pode ser realizada em uma pequena fração do mesmo, denominada amostra, que represente a realidade do lote inicial da melhor forma possível. As características de um dado material são estimadas por análises realizadas em amostras pequenas, com massas da ordem de gramas. Deste modo, a metodologia de obtenção dessas amostras é de fundamental importância a fim de minimizar os erros associadas a essas análises, para que, com base nelas, sejam realizadas as estimativas para avaliação de depósitos minerais, controle de processos em laboratórios, unidades piloto, indústrias, comercialização de produtos, entre outros (Oliveira, 2007).

A representatividade de uma amostra ocorre quando a soma da variância com a média ao quadrado do erro de amostragem possui um valor menor que o máximo estabelecido entre as partes interessadas, usualmente o produtor e o consumidor. De acordo com a Teoria de Amostragem de Pierre Gy, existe um erro de amostragem que não pode ser eliminado, chamado "erro fundamental de amostragem" e causado pela heterogeneidade constitucional do material. Este erro pode ser calculado pela "equação de Gy", que aplicável a todos os tipos de minério, mas que deve ser calibrada para cada um deles individualmente. Os testes de heterogeneidade foram desenvolvidos com o objetivo de estimar o erro fundamental de amostragem para cada tipo de minério, permitindo calcular a massa mínima da amostra representativa em cada etapa do processo, bem como definir o protocolo ótimo de amostragem e tratamento das amostras (CHIEREGATI, 2007).

\section{OBJETIVOS}

O trabalho tem como principal objetivo aumentar a confiabilidade nos resultados de amostragem para a estimativa do teor de pilhas de minério e do teor da jazida de bauxita de um empreendimento mineiro, através da calibração da Equação de Gy usando os resultados do teste de heterogeneidade.

\section{DESENVOLVIMENTO}

O erro fundamental de amostragem pode ser determinado através da Equação 1 denominada "fórmula de Gy", onde $R V$ é a variância relativa, $d$ é o diâmetro nominal dos fragmentos. Os fatores $c, f, g$ e / são tabelados para cada tipo de minério e tamanho de fragmento:

$$
R V=c * f * g * l * d^{3} *\left(\frac{1}{M S}-\frac{I}{M l}\right)
$$

O erro fundamental é estimado com base no tamanho máximo de fragmento, sendo necessária a realização de um procedimento experimental para determinar os fatores específicos para o minério em estudo. O trabalho de Pitard (1993) propõe um experimento que permite 
determinar o desvio padrão gerado pela heterogeneidade constitucional do mineral de minério, chamado teste de heterogeneidade, que correlaciona o diâmetro nominal (Equação 2) de cada fração granulométrica estudada com os respectivos fatores de heterogeneidade EST IHL calculados pela Equação 3, onde $M_{q}$ e $a_{q}$ são respectivamente a massa e o teor de cada uma das 50 amostras obtidas para cada faixa granulométrica do teste, $M_{Q}$ é a somatória de $M_{q}$ e $a_{Q}$, dado pela equação 4:

$$
\begin{aligned}
& \mathrm{d}=\sqrt[3]{\left(\mathrm{d}_{1}{ }^{3}+\mathrm{d}_{2}{ }^{3}\right) / 2} \\
& \text { EST } \mathrm{IH}_{\mathrm{L}}=g \sum \frac{\left(\mathrm{a}_{\mathrm{q}}-\mathrm{a}_{\mathrm{Q}}\right) 2}{\mathrm{aQ}^{2}} * \frac{\mathrm{M}_{\mathrm{q}}{ }^{2}}{\mathrm{M}_{\mathrm{Q}}} \\
& \mathrm{aQ}=\frac{1}{\mathrm{M}_{\mathrm{Q}}} \sum \mathrm{aq}_{\mathrm{q}} * \mathrm{M}_{\mathrm{q}}
\end{aligned}
$$

Segundo Pitard (1993), o resultado do teste de heterogeneidade permite correlacionar o diâmetro nominal de cada fração granulométrica com o fator constante de heterogeneidade constitucional EST IH $\mathrm{H}_{\mathrm{L}}$ em um gráfico di-log, obtendo-se as constantes de amostragem $k$ e $\alpha$ que representam, respectivamente, os fatores e o expoente cúbico da "Fórmula de Gy" (Equação 1), como mostra a Equação 5:

$$
R V=k * d^{\alpha} *\left(\frac{1}{M S}-\frac{I}{M l}\right)
$$

\section{PROCEDIMENTO EXPERIMENTAL}

A amostra de bauxita selecionada para o teste de heterogeneidade foi coletada na torre de amostragem da usina após o processo de beneficiamento, com massa total de $500 \mathrm{~kg}$ e fração granulométrica "-76,2 mm +1,18 mm". Uma vez transportada para o laboratório físico, a primeira etapa do processo consistiu na lavagem em peneiras vibratórias a fim de facilitar a separação granulométrica nas frações estudadas, quais sejam: "-38,1 mm +25,4 mm"; "-25,4 mm +12,7 mm"; "-12,7 mm +6,3 mm"; “-6,3 mm +1,2 mm". Após o peneiramento, cada amostra foi seca em estufa de circulação forçada na temperatura de $110^{\circ} \mathrm{C}$ durante 6 horas, garantindo assim a umidade com as seguintes massas: $100 \mathrm{~kg}$ (“-38,1 mm +25,4 mm”); $70 \mathrm{~kg}$ (“-25,4 mm +12,7 mm"); $60 \mathrm{~kg}$ (“-12,7 $\mathrm{mm}+6,3 \mathrm{~mm}$ "); $10 \mathrm{~kg}$ (“-6,3 mm +1,2 mm").

Prepararam-se duas malhas em plataformas para a distribuição dos fragmentos sendo uma de 50 quadrantes, cada um com $300 \mathrm{~mm}$ lado, e outra de 100 quadrantes cada um com $100 \mathrm{~mm}$ de lado. Os fragmentos foram distribuídos de forma homogênea nas malhas, sem superposição, de modo a garantir a equiprobabilidade de seleção. O procedimento de coleta para a faixa granulométrica mais fina ("- $6,3 \mathrm{~mm}+1,2 \mathrm{~mm}$ ") foi realizado na malha de 100 quadrados com ajuda de uma pinça, sempre com um executante e um observador, de forma alternada, da forma mais aleatória possível. Os fragmentos foram coletados um a um, aleatoriamente, de cada quadrado, de modo a gerar 50 amostras compostas por 100 fragmentos cada. Para as outras três frações não se fez necessário o uso da pinça e o procedimento foi realizado na malha de 50 quadrados 
obedecendo aos mesmos critérios para a fração mais fina. Para estas três frações, os fragmentos foram manualmente coletados um a um, aleatoriamente, de cada quadrado, de modo a gerar 50 amostras compostas por 50 fragmentos cada.

Desta forma, foram produzidas 200 amostras, sendo 50 para cada fração granulométrica que, ainda no laboratório físico, foram tratadas para posteriores análises químicas. A fração mais grossa ("-38,1 mm +25,4 mm") foi cominuída até um top size de 25,4 mm em um britador de mandíbulas, em seguida até $1,7 \mathrm{~mm}$ em um moinho de rolos, homogeneizadas e quarteadas em quarteador tipo Jones até uma massa de 40 gramas e pulverizadas em 0,106 $\mathrm{mm}$ em um moinho pulverizador. As demais frações seguiram os fluxos necessários até atingir a mesma massa e granulometria. Por fim, todas as amostras foram enviadas para o laboratório químico, onde foram determinados os teores de alumina disponível por titulação complexométrica.

\section{RESULTADOS}

Uma vez obtidos os resultados de pesagem e teores de alumina disponível para cada um dos quatro grupos de 50 amostras, foi calculado o valor de EST IH $\mathrm{L}_{\mathrm{L}}$ conforme a Equação 3, bem como os diâmetros nominais conforme a Equação 2. Conforme teoria da amostragem (GY, 1998). $O$ fator granulométrico $g$ referente a material peneirado entre duas malhas vale 0,55 . Os resultados são apresentados na Tabela 1:

Tabelas 1 - Constantes de heterogeneidade calculadas para os respectivos diâmetros nominais.

\begin{tabular}{cc}
\hline Diâmetro Nominal $(\mathbf{c m})$ & EST $\mathrm{IH}_{\mathbf{L}}$ \\
\hline 3,32 & 0,823 \\
2,09 & 0,337 \\
1,05 & 0,149 \\
0,5 & 0,008 \\
\hline
\end{tabular}

Correlacionando os resultados em um gráfico di-log (Figura 1 ) determinamos as variáveis $k$ e $\alpha$ da Equação 5 .

$$
R V=0,063 * d^{2,35} *\left(\frac{1}{M S}-\frac{I}{M l}\right)
$$

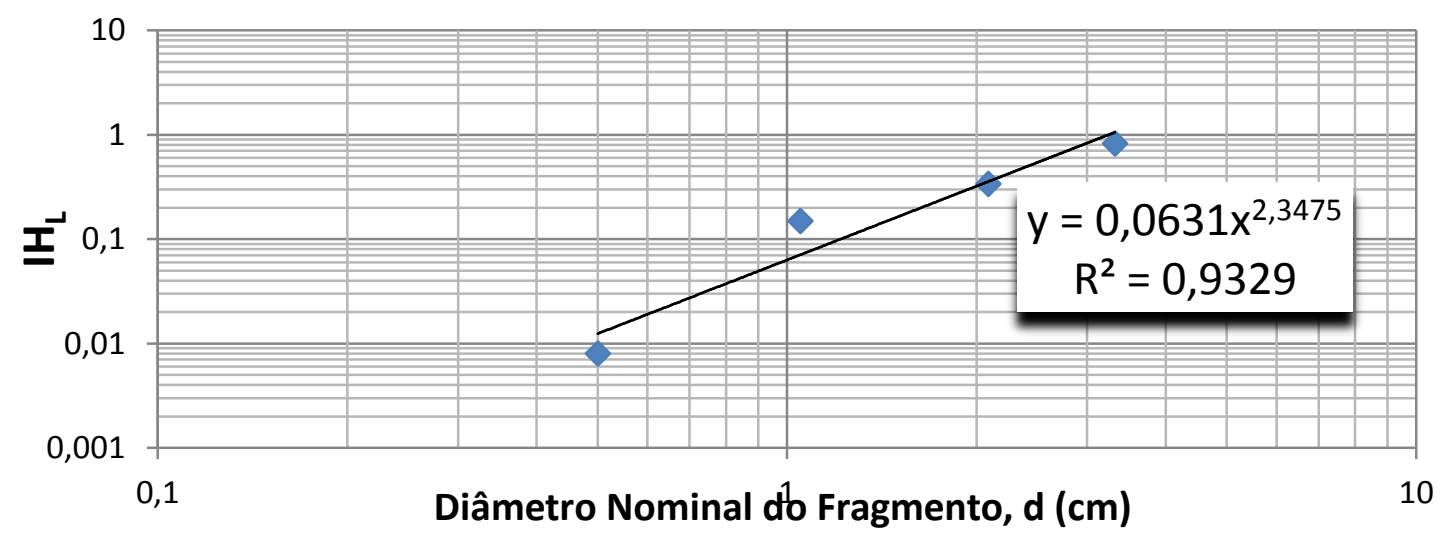

Figura 1 - Correlação gráfica entre as constantes de heterogeneidade e os diâmetros nominais. 
Uma vez conhecidas as variâncias devidas ao FSE para cada etapa da amostragem, foi proposto um novo protocolo, com as etapas críticas otimizadas (Tabela 2).

Tabela 2 - Protocolo de amostragem.

\begin{tabular}{|c|c|c|c|c|c|c|c|}
\hline Etapa & $M_{L}(g)$ & $M_{s}(g)$ & $D_{N}(\mathrm{~cm})$ & $\mathrm{IH}_{\mathrm{L}}$ & $s^{2}(F S E)$ & S(FSE) \% & S(FSE) Gy \% \\
\hline Amostragem Primária & 15000000000 & 15000 & 5 & 2,717886958 & 0,00018119 & 1,35 & 0,4 \\
\hline Britagem & 15000 & 15000 & 0,64 & 0,022181735 & 0 & 0 & 0 \\
\hline Quarteamento Primário & 15000 & 3500 & 0,64 & 0,022181735 & $4,86 \mathrm{E}-06$ & 0,22 & 0,51 \\
\hline Lavagem & 1650 & 1650 & 0,64 & 0,022181735 & 0,0000 & 0,00 & 0 \\
\hline Moagem & 1750 & 1750 & 0,2 & 0,001460326 & 0 & 0 & 0 \\
\hline Quarteamento Secundário & 1750 & 200 & 0,2 & 0,001460326 & $6,47 \mathrm{E}-06$ & 0,25 & 0,19 \\
\hline Pulverização & 200 & 200 & 0,015 & $3,41364 \mathrm{E}-06$ & 0 & 0 & 0 \\
\hline Seleção da amostra analítica & 40 & 5 & 0,015 & $3,41364 \mathrm{E}-06$ & $5,97 \mathrm{E}-07$ & 0,08 & 0,02 \\
\hline TOTAL & & & & & 0,0002 & 1,39 & 0,68 \\
\hline
\end{tabular}

\section{CONCLUSÕES}

A determinação experimental das variáveis $k$ e $\alpha$ permite a elaboração de protocolos de amostragem otimizados para o minério de alumínio, possibilitando calcular o erro em cada etapa da amostragem. Quando utilizamos a Equação 1 com os fatores de Gy (generalizados para cada tipo de minério) sem realizar a calibração experimental de $k$ e $\alpha$, podemos subestimar o erro em alguma etapa do processo. A partir dos resultados obtidos no protocolo apresentado anteriormente, observamos que a equação não calibrada de Gy estimou uma variância devido ao erro fundamental de 0,68 , isto é, quase duas vezes menor que a obtida pelo teste de heterogeneidade $(1,39)$, mostrando a importância do teste para a bauxita e o impacto que a ausência do mesmo pode causar na confiabilidade dos resultados. Podemos concluir também que, com os resultados do teste e a equação calibrada, verificou-se uma oportunidade de melhoria com a aquisição de um britador para uma cominuição inicial a 6,4 $\mathrm{mm}$ e de uma panela com capacidade de 200 g para o pulverizador, resultando assim, na redução da variância devida ao erro fundamental.

\section{REFERÊNCIAS}

1. CHIEREGATI, A. C. Reconciliação pró-ativa em empreendimentos mineiros. São Paulo, 2007, 201p. Tese de doutorado. Departamento de Engenharia de Minas e de Petróleo. Escola Politécnica da Universidade de São Paulo.

2. GY, P. 1998. Sampling for analytical purposes. 1st ed., translated by A.G. Royle, John

3. OLIVEIRA, Maria Lúcia M. de \& AQUINO, José Aury de. Amostragem. In: SAMPAIO, João Alves et al (ed.). Tratamento de minério: Práticas laboratoriais. Rio de Janeiro, CETEM/MCT, 2007, $557 p$.

4. PITARD, F.F. 1993. Pierre Gy's sampling theory and sampling practice: heterogeneity, sampling correctness, and statistical process control. 2nd ed., CRC Press, Boca Raton, Florida. 